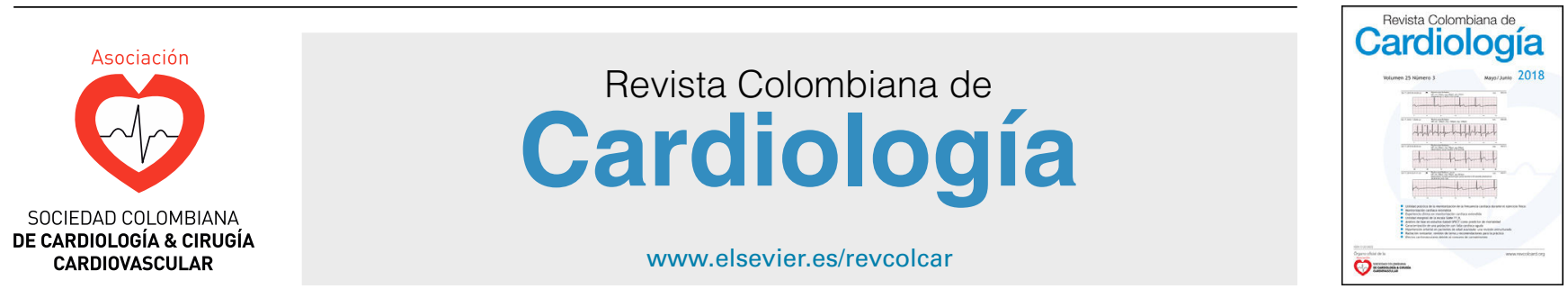

CARDIOLOGÍA DEL ADULTO - REVISIÓN DE TEMAS

\title{
Enfoque inicial de las alteraciones mediastinales: revisión de sus referencias anatómicas radiográficas
}

\section{Emmanuel Salinas Miranda*, Luisa Karen Cifuentes, Juan Gonzalo Vélez y Bibiana Andrea Pinzón}

\author{
Departamento de Radiología, Hospital universitario Fundación Santa Fe de Bogotá, Bogotá, DC, Colombia
}

Recibido el 27 de mayo de 2017; aceptado el 4 de octubre de 2017

Disponible en Internet el 5 de septiembre de 2018

\section{PALABRAS CLAVE \\ Epidemiología; \\ Cirugía; \\ Radiografía; \\ Tumores; \\ Medicina basada en la \\ evidencia}

\begin{abstract}
Resumen La sospecha de alteraciones mediastinales, siempre incluye en su estudio inicial, la radiografía de tórax. La identificación de las alteraciones mediastinales en la radiografía es una prioridad. El conocimiento de las referencias mediastinales y la identificación de sus alteraciones, permite sospechar una patología propia de cada uno de los espacios mediastinales. Cuando se tiene en cuenta la semiología de las lesiones mediastinales, su ubicación y las tres patologías más frecuentes, se incrementa la posibilidad de tener un diagnóstico etiológico ${ }^{1}$. Este es un artículo de revisión, a partir de una búsqueda detallada de la literatura, en el que se estudian las referencias mediastinales radiológicas, con énfasis en los datos epidemiológicos de cada una de ellas.

(c) 2018 Sociedad Colombiana de Cardiología y Cirugía Cardiovascular. Publicado por Elsevier España, S.L.U. Este es un artículo Open Access bajo la licencia CC BY-NC-ND (http:// creativecommons.org/licenses/by-nc-nd/4.0/).
\end{abstract}

\section{KEYWORDS \\ Epidemiology; \\ Surgery; \\ Radiography; \\ Tumours; \\ Evidence-based \\ medicine}

Initial approach to changes in the mediastinum: A review of the radiographic anatomical references

\begin{abstract}
The initial study in the suspicion of changes in the mediastinum should always include a chest $x$-ray. The identification of these changes on the $x$-ray is a priority. Knowledge of the mediastinal references and the identification of their changes can lead to the suspicion of a disease common to each of the mediastinal spaces. When the semiology of the mediastinal lesions is taken into account, as well as its location and the three most common diseases, the possibility of making an aetiological diagnosis increases. This is a review article, using a detailed literature search, in which the radiological mediastinal references are studied, with an emphasis on the epidemiological data of each one of them.

(C) 2018 Sociedad Colombiana de Cardiología y Cirugía Cardiovascular. Published by Elsevier España, S.L.U. This is an open access article under the CC BY-NC-ND license (http:// creativecommons.org/licenses/by-nc-nd/4.0/).
\end{abstract}

\footnotetext{
* Autor para correspondencia.

Correo electrónico: emmanuels7@gmail.com (E. Salinas Miranda).
} 


\section{Introducción}

El mediastino es un compartimento anatómico intratorácico extrapleural, situado en el centro del tórax, entre ambos pulmones, detrás del esternón y las uniones condrocostales y delante de los cuerpos vertebrales y las costillas. En su aspecto inferior se encuentra limitado por el músculo diafragma y en su aspecto superior por el estrecho cervicotorácico.

En la radiografía de tórax, las referencias mediastinales pueden ser producto de la continuidad de las estructuras mediastinales, pulmonares o vertebrales cuando son atravesadas de forma tangencial por el haz de rayos $x$ o pueden ser un efecto visual como las bandas Mach de las que se hablará más adelante. Estas siluetas pueden dividirse en líneas, bandas o interfaces (fig. 1) ${ }^{1,2}$.

La ausencia, engrosamiento o desplazamiento de una o varias de las líneas, bandas o interfaces mediastinales puede significar una lesión mediastinal; sin embargo, las condiciones técnicas de la radiografía y las variaciones anatómicas, también modifican la frecuencia y la forma en la que son observadas estas siluetas mediastinales o sus alteraciones. Las líneas que más se visualizan son la banda paratraqueal y la línea paraespinal del lado derecho ${ }^{2}$. El resto de las referencias mediastinales son visualizadas en porcentajes variables en la radiografía de tórax (tabla 1).

El primer paso para abordar las masas mediastinales en la radiografía de tórax, es sospechar su origen mediastinal. Las características radiográficas de una lesión que orientan a un origen mediastinal son: la relación íntima de la masa con las estructuras mediastinales, las márgenes lisas y nítidas y la formación de ángulos obtusos entre la masa y el campo pulmonar $^{1,3}$ (fig. 2).

El efecto íntimo con las estructuras mediastinales se infiere a partir de la alteración y desplazamiento de las estructuras mediastinales adyacentes a la lesión, como la tráquea, los bronquios principales o el corazón (fig. 3).

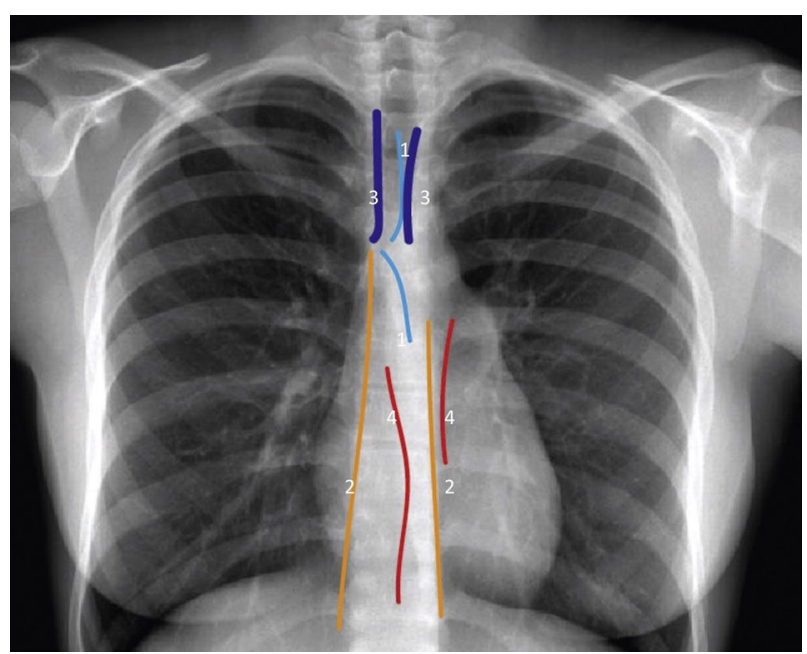

Figura 1 Líneas, bandas e interfaces del mediastino Esquema de radiografía de tórax con líneas, bandas e interfaces. Representación de líneas de unión superior-posterior e inferioranterior (1); Líneas paraespinales (2), bandas paratraqueales (3) e interfaces azygo-aesofáceas y paraórticas (4).
Tabla 1 Frecuencia de visualización de las líneas, bandas e interfaces mediastinales

\begin{tabular}{ll}
\hline Línea de unión anterior & $24 \%$ \\
Línea de unión posterior & $32 \%$ \\
Banda paratraqueal derecha & $97 \%$ \\
Banda paratraqueal izquierda & $31 \%$ \\
Línea paraespinal derecha & $23 \%$ \\
Línea paraespinal izquierda & $41 \%$ \\
Receso paraórtico izquierdo & $40 \% \wedge$ \\
Interfaz ácigo - esofágica & $5 \%$ \\
Ventana aortopulmonar & $90 \%$ \\
\hline
\end{tabular}

* Cálculo aproximado de Biemans JM ${ }^{43} \wedge$ Calculado de Dobson
MJ 44

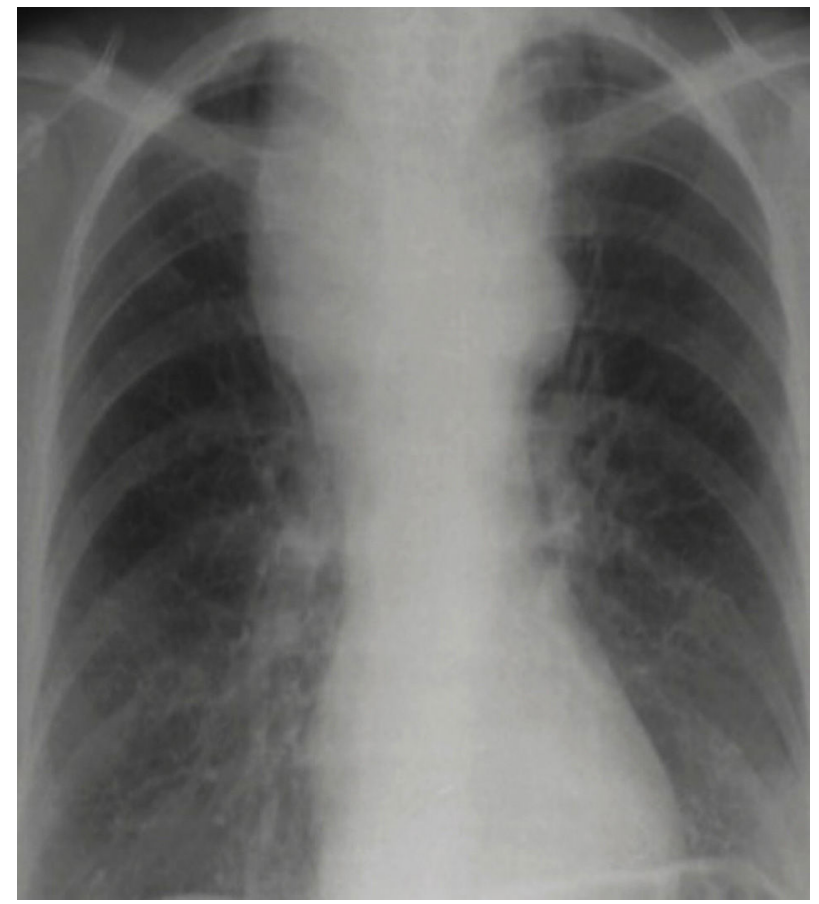

Figura 2 Sospecha de una masa mediastinal

Radiografía de tórax donde se identifica una masa en el tercio superior del tórax, que muestra un efecto íntimo con estructuras mediastínicas. La masa condiciona desplazamiento de la tráquea. Presenta márgenes bien definidos y agudos con ángulos obtusos. Este paciente tiene un diagnóstico histológico de carcinoma tiroideo.

Todas las lesiones mediastinales tienen características individuales, sin embargo, algunas de ellas, se pueden solapar, especialmente en la radiografía de tórax, la cual presenta gran limitación respecto a la tomografía al tratarse de una proyección única, la mayoría de veces, solamente anteroposterior (tabla 2).

\section{Metodología}

Se realizó una revisión de las referencias radiológicas mediastinales, con énfasis en sus características por imágenes y su contexto epidemiológico. Se realizó una búsqueda detallada de la literatura en las bases de datos médicas disponibles según términos «MeSH» y filtros específicos. Los 


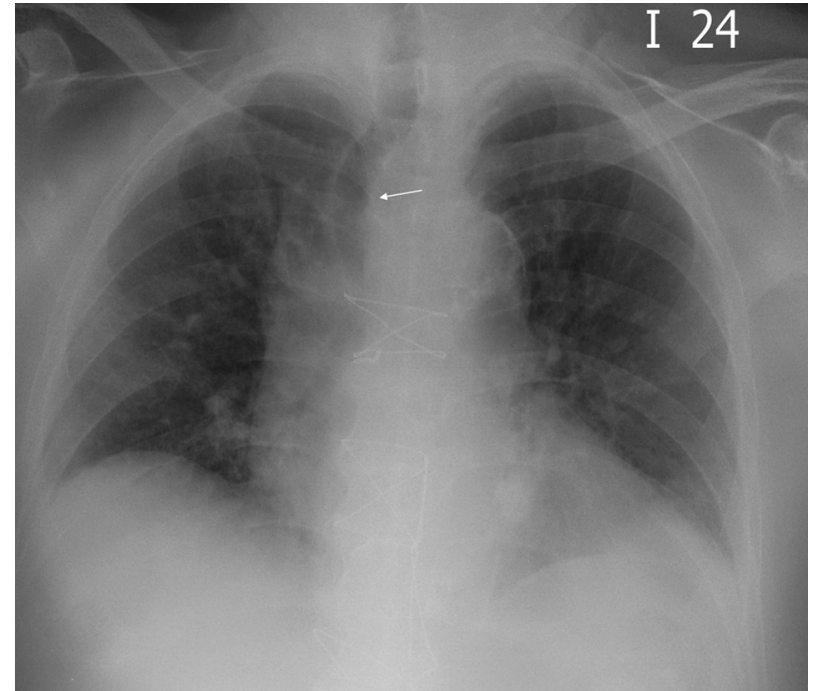

Figura 3 Distorsión de la anatomía

Radiografía de tórax que muestra efecto íntimo con estructuras mediastínicas. Distorsión de la posición de las estructuras de la línea media. Nótese la desviación de la tráquea a la derecha (flecha).

artículos que resultaron de esta búsqueda fueron analizados y seleccionados para efectuar la revisión. Se utilizaron casos representativos de nuestra institución y se diseñaron esquemas para ejemplificar las referencias mediastinales.

\section{Efecto Mach}

El efecto Mach representa un fenómeno de realce de los bordes de las estructuras mediastinales en la radiografía. En este efecto los bordes de los objetos más radiopacos (blancos), aparecen más radiolúcidos (negros) por lo que se crea una sombra falsa ${ }^{4}$. De esta forma, alrededor de los objetos más radiopacos, como las vértebras y el corazón, que se encuentran junto a objetos más radiolúcidos, como los campos pulmonares, aparece una sombra oscura (radiolúcida). Este efecto se presenta con más frecuencia adyacente a las estructuras cóncavas como el corazón. El efecto Mach permite visualizar las líneas paraespinales, ya que la presencia de éstas no se da por la interfaz del pulmón con los tejidos paraespinales, puesto que los cuerpos vertebrales son más radiopacos que los campos pulmonares adyacentes ${ }^{5}$ (fig. 4). El efecto Mach también explica el pseudoneumomediastino, el cual es la falsa impresión de pneumomediastino alrededor de la silueta cardiaca ${ }^{6}$.

\section{Compartimientos y anatomía mediastinal}

La anatomía mediastinal se puede dividir en partes basándose en su relación con el pericardio fibroso. El mediastino superior se encuentra por encima del nivel superior del pericardio y plano de Ludwig, el cual se define como una línea horizontal que va de la articulación manubrioesternal al platillo vertebral inferior de $\mathrm{T}^{7}$. El mediastino inferior se encuentra por debajo del plano de Ludwig. El mediastino anterior es el compartimiento anterior al pericardio. El mediastino medio se encuentra dentro del pericardio y el mediastino posterior es el compartimiento posterior al pericardio (tabla 3). Cada uno de los compartimientos mediastinales contiene estructuras propias, las cuales son susceptibles de alteraciones.

Existen distintas clasificaciones radiológicas para dividir los compartimientos del mediastino, cada una con variaciones discretas en los límites entre cada uno de ellos. Las clasificaciones más utilizadas son los modelos de tres y cuatro compartimientos. Están el modelo Felson, que es el mismo que la clasificación de Zylak ${ }^{8}$, el modelo de Fraser y Paré ${ }^{9}$, la clasificación de Burkell ${ }^{10}$, el modelo propuesto por la Asociación japonesa para la investigación del timo $(\mathrm{JART})^{10}$ y el modelo anatómico. El modelo de la JART y la clasificación anatómica dividen el mediastino en cuatro compartimientos, mientras que la clasificación de Felson y de Burkell lo dividen en tres. Recientemente se ha propuesto una clasificación alternativa, denominada ITMIG (Grupo Internacional de Interés de Malignidad Tímica), basada en imágenes de cortes transversales de tomografía computarizada; sin embargo, su revisión excede los objetivos de este artículo $^{11}$.

El modelo de tres compartimientos (Modelo de Felson) es el que se utilizará a lo largo de esta revisión por ser el que permite un abordaje más simple en la radiografia de tórax (fig. 5)

El modelo de Felson, que es el mismo que la clasificación de Zylak, utiliza como referencia la radiografía lateral de tórax. El mediastino anterior y medio están separados por una línea que se extiende a lo largo del dorso de la silueta del corazón y del aspecto anterior de la tráquea. El mediastino posterior se encuentra entre la línea trasera de la tráquea y una línea ubicada $1 \mathrm{~cm}$ posterior al margen anterior de los cuerpos vertebrales.

\section{Semiología del mediastino según sus líneas, bandas e interfaces}

Las referencias radiológicas de la anatomía mediastinal en la radiografía de tórax son las líneas, las bandas y las interfaces.

Una línea es una opacidad longitudinal de no más de 1$2 \mathrm{~mm}$ de ancho. Son las líneas de unión pleural anterior y posterior y las líneas paraespinales derecha e izquierda. Se forman de la aposición estrecha de las pleuras parietal y visceral de ambos pulmones, en el caso de las líneas de unión, o de la aposición de las dos capas de la pleura a los márgenes laterales de los cuerpos vertebrales, en el caso de las líneas paraespinales. Estas últimas también pueden explicarse por el efecto Mach.

Una banda es una opacidad longitudinal de $2-5 \mathrm{~mm}$ de ancho. En el mediastino corresponden a las bandas paratraqueales. Se forman de la aposición de las pleuras parietales de los lóbulos superiores con las paredes laterales de la tráquea.

Una interfaz se forma de la aposición de dos tejidos de diferentes densidades, como los pulmones y el corazón. La interfaz acigo-esofágica y la interfaz de la aorta descendente son las referencias anatómicas más importantes.

El desplazamiento de una línea mediastinal, el ensanchamiento de una banda o el contorno anormal de una interfaz son signos importantes de patología mediastinal (fig. 6). 
Tabla 2 Características radiográficas de las lesiones mediastinales

\begin{tabular}{|c|c|c|}
\hline Tumor & Localización mediastinal & Características radiográficas \\
\hline Timoma & Anterior & $\begin{array}{l}\text { Masa lobulada de contornos bien definidos } \\
\text { Mediastino anterosuperior } \\
\text { Anterior al botón aórtico. }\end{array}$ \\
\hline Timolipoma & Anterior & $\begin{array}{l}\text { Ensanchamiento mediastinal difuso. } \\
\text { Márgenes claras con el pulmón } \\
\text { No hay efecto de masa significativo. }\end{array}$ \\
\hline Teratoma & Anterior & $\begin{array}{l}\text { Opacidad en el mediastino anterior. } \\
\text { Calcificaciones }\end{array}$ \\
\hline Bocio retroesternal & Anterior & $\begin{array}{l}\text { Masa lobulada, homogénea } \\
\text { Mediastino anterosuperior } \\
\text { Continuidad con las estructuras cervicales. }\end{array}$ \\
\hline Lipoma & Anterior & $\begin{array}{l}\text { Masa lobulada homogénea en mediastino } \\
\text { anterosuperior. }\end{array}$ \\
\hline $\begin{array}{l}\text { Tumores de las células } \\
\text { germinales }\end{array}$ & Anterior & $\begin{array}{l}\text { Masa lobulada o redonda. } \\
\text { Bien definida. } \\
\text { Puede estar calcificada (huesos o dientes). } \\
\text { Masa lobulada de gran volumen (seminoma) }\end{array}$ \\
\hline Quiste broncogénico & Medio & $\begin{array}{l}\text { Masas redonda, bien definida, homogénea. } \\
\text { Densidad radiolúcida o apariencia sólida. }\end{array}$ \\
\hline $\begin{array}{l}\text { Extensión de tumores } \\
\text { broncogénicos }\end{array}$ & Medio & $\begin{array}{l}\text { Linfadenomegalias } \\
\text { Ensanchamiento mediastinal } \\
\text { Masa lobulada, heterogénea, de bordes mal } \\
\text { definidos. } \\
\text { Masa o nódulo en el parénquima pulmonar. }\end{array}$ \\
\hline Linfoma & Anterior y medio & Adenomegalias \\
\hline Tumores neurogénicos & Posterior & $\begin{array}{l}\text { Lesiones esféricas, muy bien definidas. } \\
\text { Adyacentes a los cuerpos vertebrales. } \\
\text { Erosión de las costillas adyacentes. } \\
\text { Erosión de los forámenes de conjugación o las } \\
\text { vértebras. } \\
\text { Obliteración de las líneas paraespinales. }\end{array}$ \\
\hline Aneurisma aórtico & Posterior & $\begin{array}{l}\text { Masa posterior en continuidad con el arco aórtico. } \\
\text { Calcificaciones curvilíneas ateromatosas }\end{array}$ \\
\hline Infecciones vertebrales & Posterior & $\begin{array}{l}\text { En estadios tempranos puede no haber hallazgos. } \\
\text { Destrucción osteolítica de la vértebra. } \\
\text { Colapso vertebral anterior. } \\
\text { Prominencia de los tejidos blandos } \\
\text { prevertebrales. }\end{array}$ \\
\hline Hematopoyesis extramedular & Posterior & $\begin{array}{l}\text { Masas de márgenes bien definidos, delineados. } \\
\text { Arcos costales trabeculados y costillas } \\
\text { ensanchadas. } \\
\text { Más frecuentemente en arcos costales inferiores. }\end{array}$ \\
\hline Meningocele & Posterior & $\begin{array}{l}\text { Opacidades paravertebrales bien definidas } \\
\text { Márgenes bien definidas o lobuladas. }\end{array}$ \\
\hline Hematomas prevertebrales & Posterior & $\begin{array}{l}\text { Borramiento de las líneas paraspinales. } \\
\text { Puede o no existir ensanchamiento mediastinal } \\
\text { Incremento de la línea interpeduncular. } \\
\text { Fractura/lesión vertebral }\end{array}$ \\
\hline Quistes neuroentéricos. & Posterior & $\begin{array}{l}\text { Lesión homogénea hiperdensa de márgenes bien } \\
\text { definidas } \\
\text { Se pueden asociar a lesiones vertebrales en un } \\
\text { nivel diferente. }\end{array}$ \\
\hline Sarcomas & Posterior & $\begin{array}{l}\text { Masas de gran tamaño mal definidas } \\
\text { Erosiones óseas }\end{array}$ \\
\hline
\end{tabular}




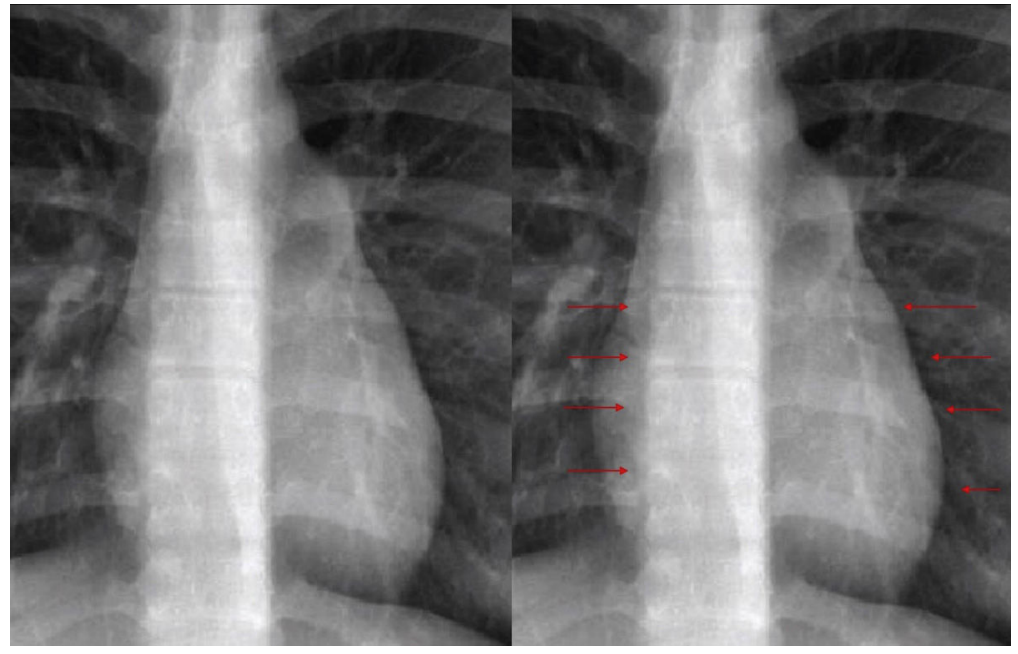

Figura 4 Efecto mach

El efecto mach es un efecto óptico entre áreas de densidad ligeramente diferente. Puede imitar un pneumo-mediastino. En estas imágenes se muestran la línea paraspinal derecha y la silueta cardiaca izquierda, las cuales son líneas mach.

Tabla 3 División anatómica mediastinal con sus límites y las estructuras que contienen

\begin{tabular}{|c|c|c|}
\hline División & Límites & Estructuras normales \\
\hline Anterior & $\begin{array}{l}\text { Anterior: esternón Posterior: margen } \\
\text { anterior del pericardio, aorta y vasos } \\
\text { braquiocefálicos. }\end{array}$ & $\begin{array}{l}\text { Timo } \\
\text { Nódulos linfáticos } \\
\text { Grasa } \\
\text { Vasos mamarios internos. }\end{array}$ \\
\hline Medio & $\begin{array}{l}\text { Anterior: margen posterior de la división } \\
\text { anterior Posterior: margen anterior de la } \\
\text { división posterior }\end{array}$ & $\begin{array}{l}\text { Corazón y pericardio } \\
\text { Aorta ascendente y transversa } \\
\text { Vasos braquiocefálicos } \\
\text { Vena cava superior e inferior Vasos pulmonares } \\
\text { principales } \\
\text { Tráquea y bronquios principales Nódulos } \\
\text { linfáticos } \\
\text { Grasa }\end{array}$ \\
\hline Posterior & $\begin{array}{l}\text { Anterior: borde posterior del pericardio. } \\
\text { Posterior: fascia prevertebral y ligamento } \\
\text { longitudinal anterior. } \\
\text { * Los márgenes laterales de todos los } \\
\text { compartimientos pulmonares son las } \\
\text { pleuras parietales. }\end{array}$ & $\begin{array}{l}\text { Aorta descendente } \\
\text { Esófago } \\
\text { Ducto torácico } \\
\text { Vena ácigos o hemiácigos } \\
\text { Nervios autonómicos } \\
\text { Nódulos linfáticos } \\
\text { Grasa }\end{array}$ \\
\hline
\end{tabular}

\section{Mediastino anterior}

Las alteraciones del mediastino anterior se visualizan usualmente como alteración de la línea de unión anterior, la presencia del signo de superposición del hilio y, en el caso de una masa, su continuidad con el diafragma y la ausencia de su interfaz con el pulmón (tabla 4).

\section{Alteración de la línea de unión anterior}

Esta línea se localiza posterior al esternón, en sus dos tercios superiores y tiene una orientación oblicua de derecha a izquierda y cefálica a caudal.
Es visualizada en el 24 al 50\% de las radiografías de tórax y se observa más fácilmente en pacientes con neumotórax o con enfisema por la hiperaireación del pulmón (fig. 7). Puede desaparecer o engrosarse de forma focal o difusa por la presencia de masas mediastinales anteriores. Sus contornos se pueden alterar por las lesiones propias del mediastino anterior como el bocio endotorácico, el timoma y la mediastinitis ${ }^{3}$.

En el adulto, las masas del mediastino anterior presentan el mayor riesgo de malignidad. Aproximadamente el $56 \%$ de todas las lesiones del mediastino anterior son malignas ${ }^{12,13}$. Las masas más frecuentes en el mediastino anterior son las masas tímicas y las de la glándula tiroides. Estas masas alteran el contorno de las líneas de unión anterior (fig. 8). 


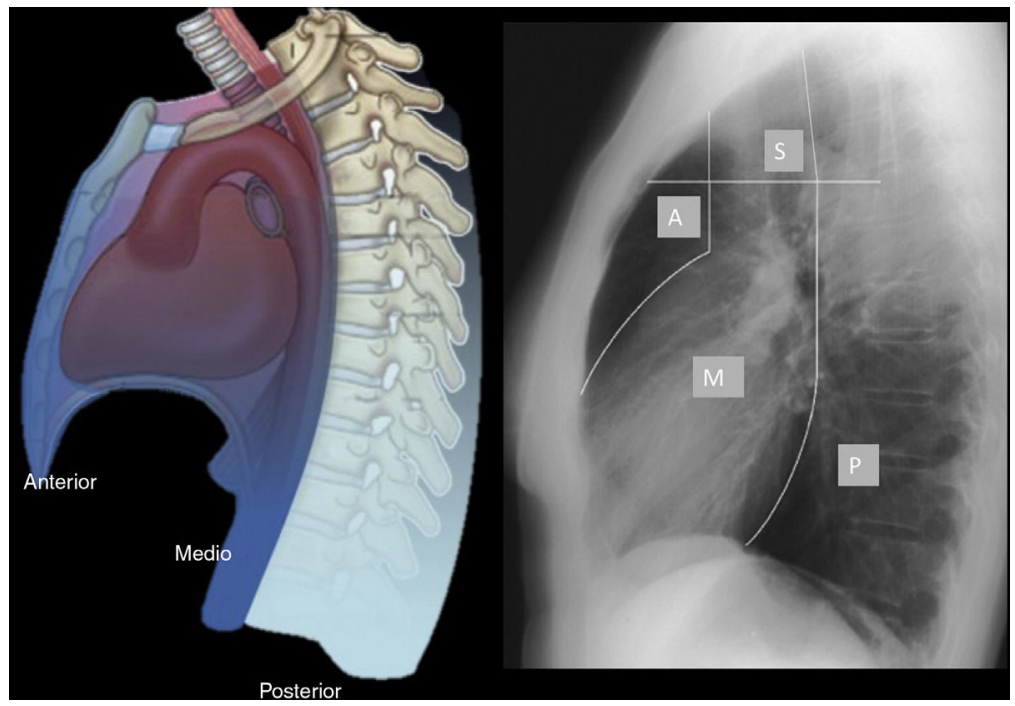

Figura 5 Modelo de Felson.

El modelo de Felson (izquierda), es una clasificación radiológica y divide el mediastino en 3 compartimientos. Este modelo utiliza como referencia la radiografía lateral de tórax. En la clasificación de la derecha, diferente al modelo Felson hay mediastino superior e inferior.

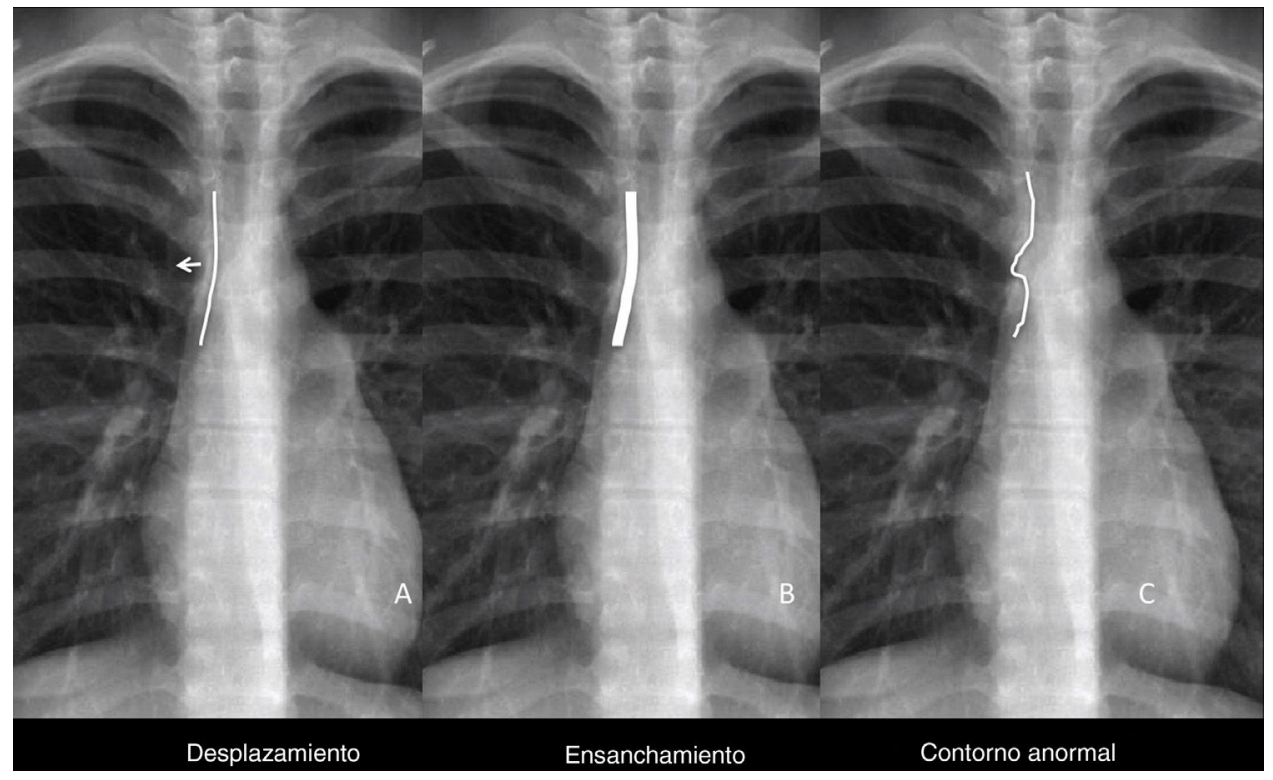

Figura 6 Esquemas que representan alteraciones en la banda paratraqueal derecha.

A. desplazamiento B. ensanchamiento C. contorno anormal. Esos cambios son signos importantes de patología mediastínica.

Tabla 4 Signos de alteración del mediastino anterior

Alteración de la línea de unión anterior Signo de superposición del hilio

Signo cervicotorácico

Signo de la silueta

Signo de la convergencia hiliar

\section{Signo de superposición del hilio}

Es útil para distinguir una masa hiliar de una no hiliar. Permite ubicar una masa en el mediastino anterior o posterior.
Si se logran visualizar los vasos hiliares a través de la masa, significa que ésta no nace del hilio y por tanto estará localizada en el mediastino anterior o posterior. La mayoría de estas masas estarán localizadas en el mediastino anterior ${ }^{14}$ (fig. 9).

\section{Signo cervicotorácico}

El mediastino anterior termina en el aspecto superior de las clavículas y el posterior finaliza por encima del nivel de éstas. Cuando una masa se proyecta por encima de las clavículas lo más probable es que se encuentre localizada 


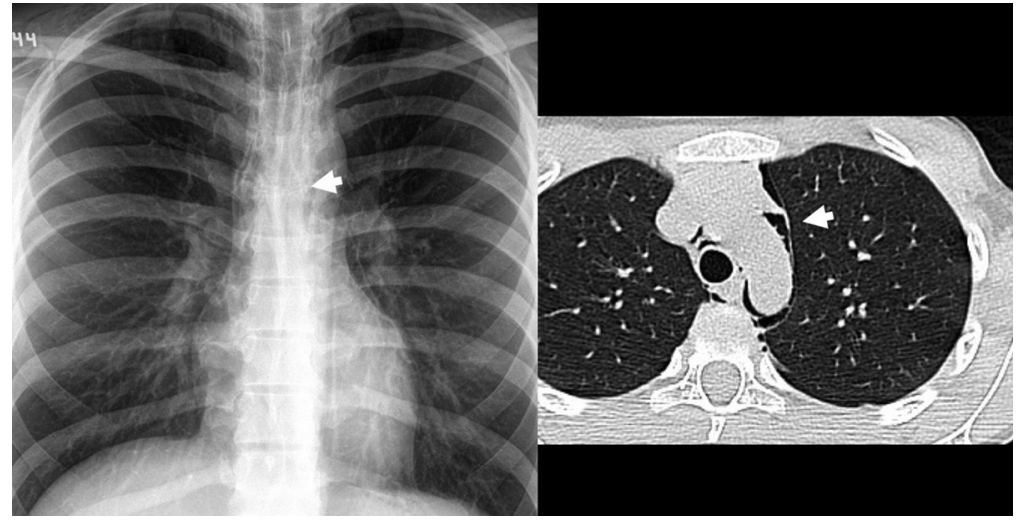

Figura 7 Aumento en la visualización de la línea anterior.

Antecedente de trauma cerrado cervicotorácico. La radiografía de tórax muestra signos de neumomediastino y enfisema subcutáneo en región supraclavicular. Se puede identificar con más facilidad la línea de unión anterior relacionado con el neumomediastino(flecha).

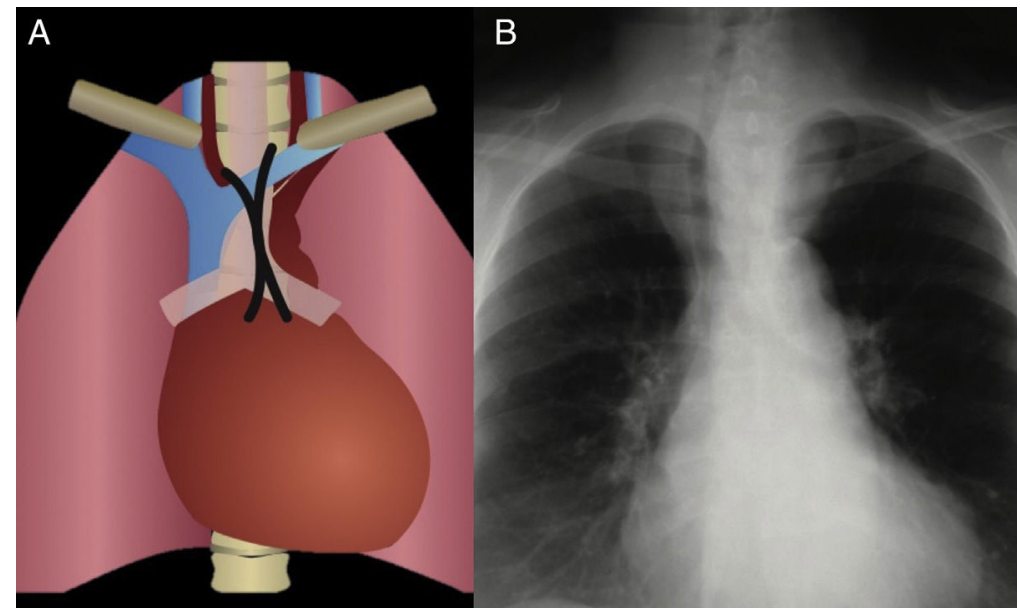

Figura 8 Distorsión de la línea de unión anterior

A. Esquema que muestra la localización de la línea de unión anterior normal B. Masa en el tercio superior del mediastino anterior con borramiento de la línea de unión anterior, desplazamiento de la tráquea a la derecha y clara interfase con el pulmón, representando una masa mediastinal. El paciente es diagnosticado con bocio endotorácico.

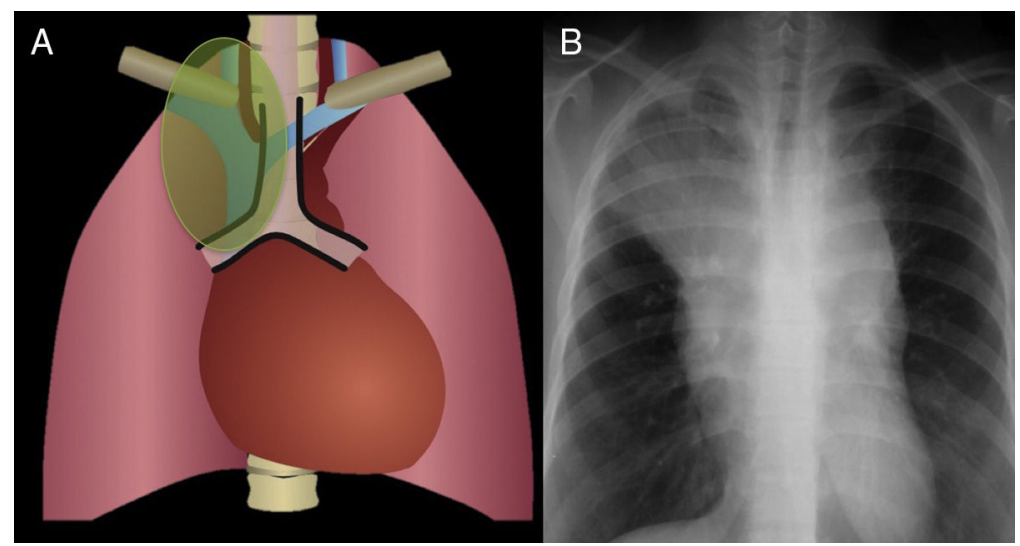

Figura 9 Signo de superposición del hilio

A. Esquema que muestra las líneas parahiliares. B. Radiografía de tórax que muestra una masa de bordes bien definidos localizada en el tercio superior del mediastino, signo de superposición del hilio y desaparición de la línea de unión anterior. El paciente fue diagnosticado con carcinoma tímico. El signo de superposición del hilio puede ayudar en la diferenciación de masas mediastinales anteriores o posteriores de masas del mediastino medio. Las masas mediastinales anteriores o posteriores se sobreponen al hilio sin desplazarlo. 


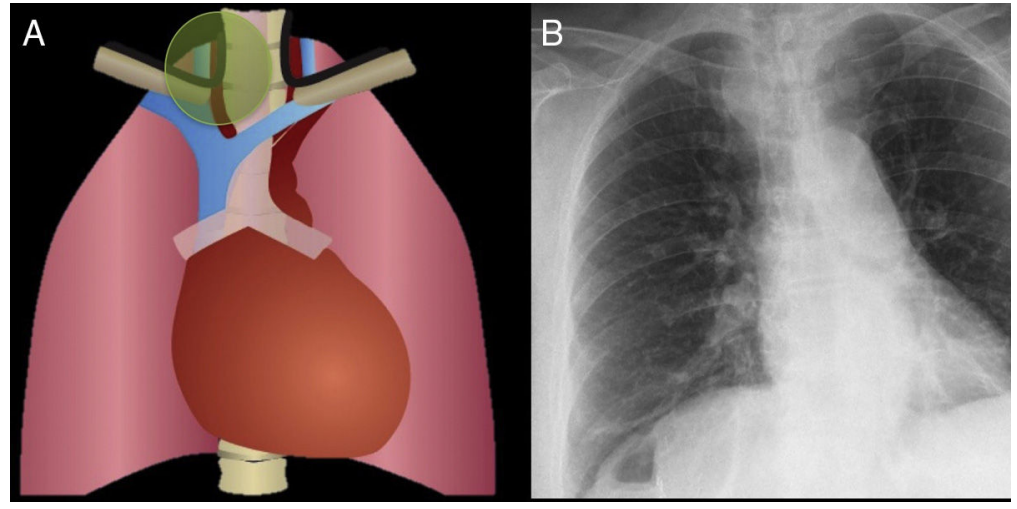

Figura 10 Masa mediastinal posterior

Masa mediastinal proyectándose sobre la clavícula derecha, con clara interfase con el pulmón y márgenes bien definidos. Se confirmó el bocio endotorácico. Cuando una masa se proyecta por encima de las clavículas, es probable que esté situada en el mediastino posterior o en estructuras cervicales.

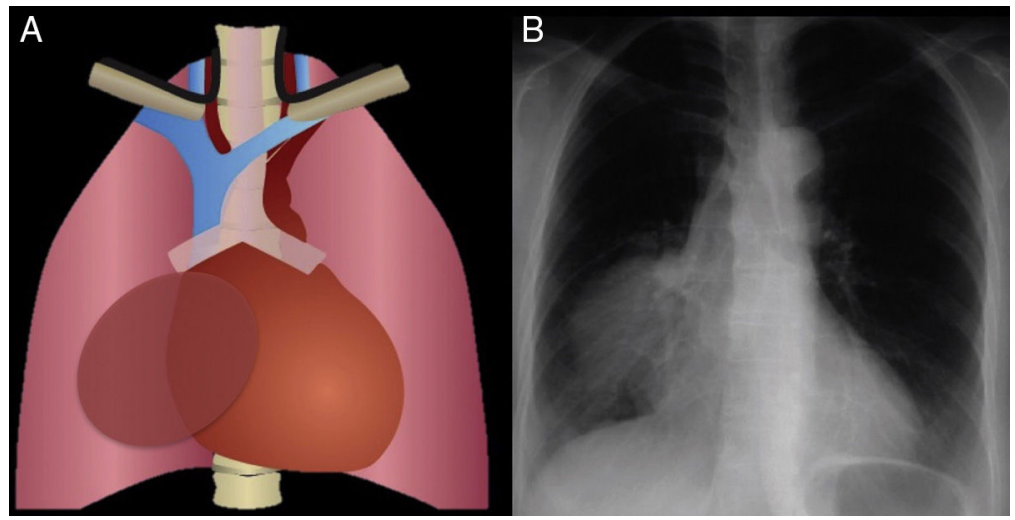

Figura 11 Signo de la silueta

Masa homogénea parahilar derecha, de bordes bien definidos, en contacto con el diafragma y haciendo signo de la silueta con el mediastino en un paciente con carcinoma de timo.

en el mediastino medio o posterior o en el cuello ${ }^{15}$. Adicionalmente, las masas supraclaviculares que no se encuentran dentro del mediastino, no tienen una interfaz clara con el pulmón y sus márgenes no son bien definidas, contrario a las masas mediastinales supraclaviculares ubicadas en el mediastino medio o posterior (fig. 10).

\section{Signo de la silueta}

Este signo consiste en la pérdida de los bordes de las estructuras de la misma densidad que se encuentran en contacto. Las masas del mediastino anterior en contacto con el borde anterior del diafragma o el pericardio, tendrán bordes poco distinguibles. Por tanto, las masas del mediastino anterior usualmente presentan el signo de la silueta. En la patología del mediastino anterior, las lesiones que presentan este signo son los lipomas, los quistes pericárdicos y las hernias de Morgani (fig. 11).

\section{Mediastino medio}

Las lesiones del mediastino medio se pueden manifestar como la obliteración de la ventana aorto-pulmonar o el
Tabla 5 Signos de alteración del mediastino medio

Obliteración de la ventana aorto-pulmonar

Ensanchamiento de las bandas paratraqueales

Ensanchamiento mediastinal

Linfadenomegalias

Desviación de la tráquea

Alteración en la interfaz de la subclavia izquierda.

ensanchamiento de las bandas paratraqueales. La presencia de linfadenomegalias y el ensanchamiento mediastinal son otros hallazgos que también orientan a las alteraciones del mediastino medio (tabla 5).

\section{Obliteración de la ventana aorto-pulmonar}

La ventana aorto - pulmonar es la concavidad formada entre el arco aórtico y la arteria pulmonar izquierda. Se encuentra delimitada lateralmente por la pleura parietal y medialmente por el ligamento arterioso. Su apariencia normal es una concavidad. Es una referencia anatómica visible en la mayoría de las radiografías de tórax. Se puede tornar convexa ante la presencia de linfadenopatías, el aumento del 


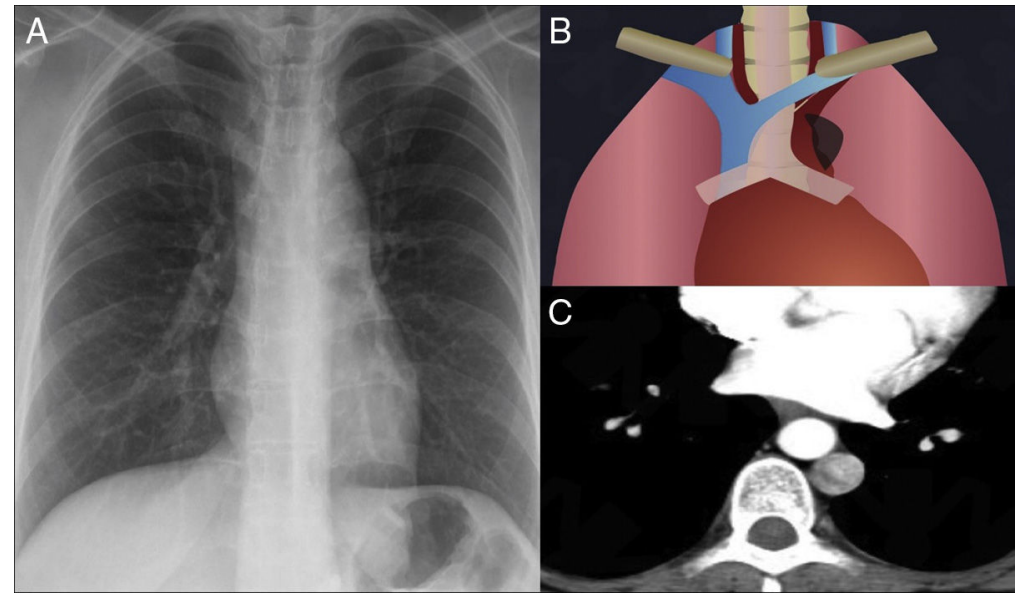

Figura 12 Ventana aortopulmonar

Radiografía de tórax (A) e ilustración (B) que muestran el contorno convexo de la ventana aortopulmonar, el cual es considerado anormal. La tomografía (C) revela la presencia de la vena cava superior izquierda como variante anatómica. Las masas mediastínales del compartimento anterior o los tumores de la vaina nerviosa del nervio laríngeo recurrente y del nervio vago izquierdo también pueden alterar el contorno de la ventana aortopulmonar.

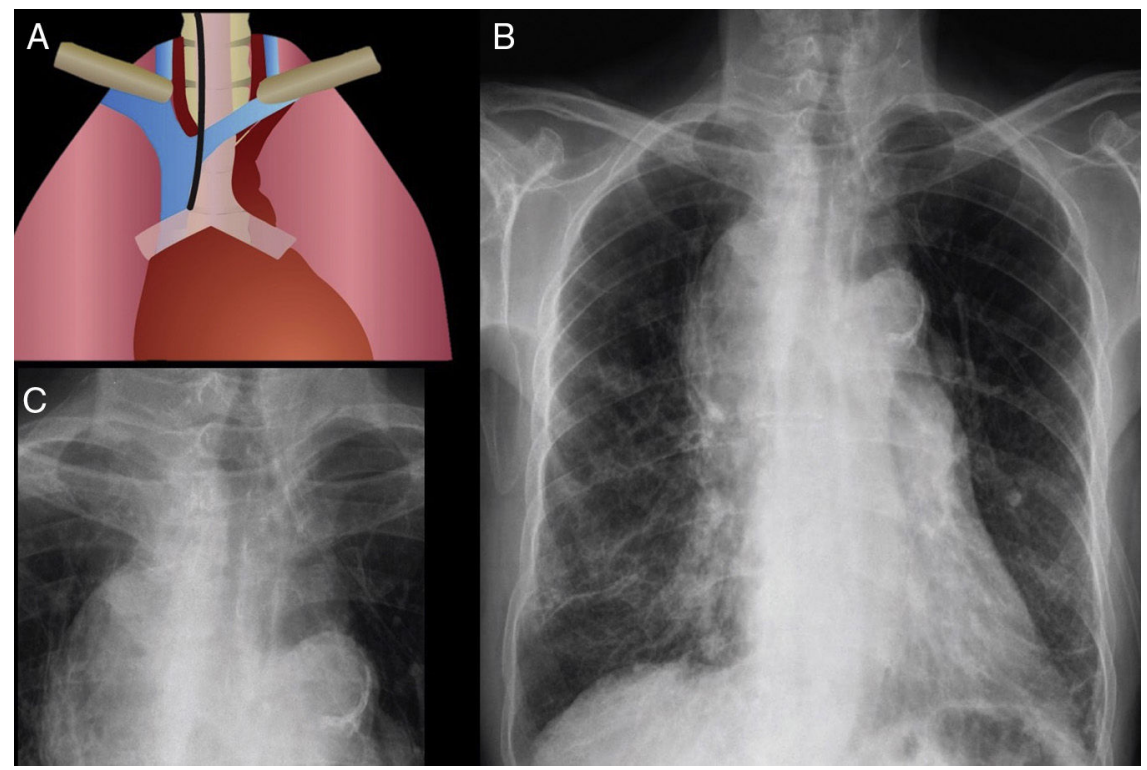

Figura 13 Banda paratraqueal derecha.

Engrosamiento de ambas bandas paratraqueales, predominantemente la banda derecha, sugestivo de enfermedad del mediastino medio. Se identifica linfadenopatía paratraqueal asociada. Se confirmó carcinoma pulmonar de células pequeñas.

tamaño del ductus arterioso y los aneurismas de la aorta. Las masas mediastinales del compartimiento, los tumores de la vaina nerviosa del nervio laríngeo recurrente y del nervio vago izquierdos también pueden alterar el contorno de la ventana aorto-pulmonar (fig. 12).

Ante la parálisis diafragmática o parálisis de la cuerda vocal izquierda se debe evaluar la ventana aortopulmonar para descartar lesiones tumorales del nervio laríngeo y del nervio vago ${ }^{1,2,16}$.

\section{Ensanchamiento de la banda paratraqueal derecha}

Es uno de los referentes anatómicos que más se visualiza en la radiografía de tórax, y llega a estar hasta en el $97 \%$ de estas ${ }^{14}$. La banda paratraqueal derecha tiene un grosor de 1 - $4 \mathrm{~mm}$, se encuentra formada por la pared traqueal, el tejido mediastinal y la pleura adyacente del pulmón derecho; tiene, además, una estrecha relación con la vena ácigos (fig. 13). 


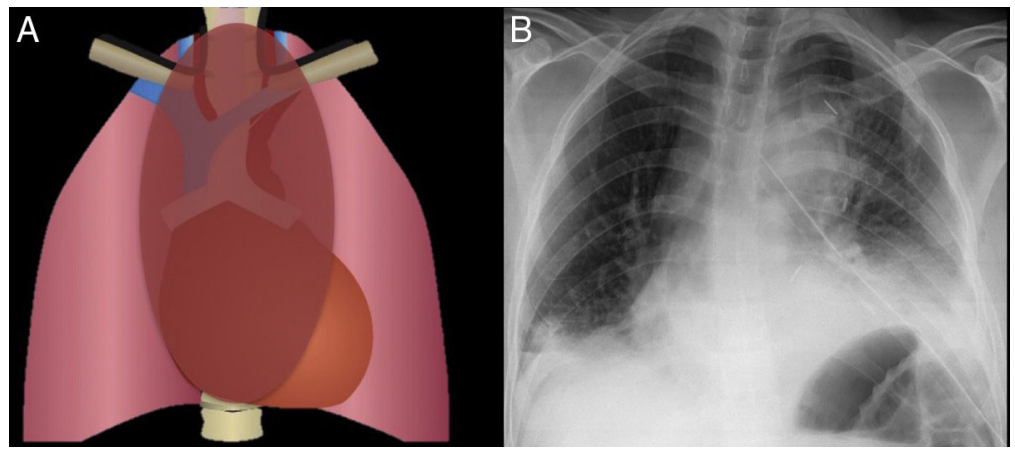

Figura 14 Ensanchamiento mediastinal

Engrosamiento de las bandas paratraqueales, predominantemente banda derecha, sugestivo de enfermedad de mediastino medio. Linfadenopatía paratraqueal asociada. Se confirma carcinoma pulmonar de células pequeñas.

El engrosamiento de la banda paratraqueal puede deberse a lipomatosis mediastinal, linfadenomegalias, masas traqueales o hematomas mediastinales. Ante el antecedente de trauma una banda paratraqueal derecha de grosor normal, disminuye la posibilidad de hematoma mediastinal y lesión de grandes vasos ${ }^{17}$.

\section{Línea paratraqueal izquierda}

Se forma del contacto entre el pulmón izquierdo, la pared traqueal izquierda y los tejidos blandos adyacentes; se sitúa sobre el arco aórtico, bajo el estrecho torácico superior. Puede ser una línea o una banda y su visualización es poco común, siendo apreciable solo en un 21 a $31 \%$ de las radiografías ${ }^{2,18,19}$; por este motivo, su ensanchamiento puede ser difícil de apreciar en las radiografías de tórax.

De forma similar a la banda paratraqueal derecha, la presencia de osteofitos, lipomatosis medistinal o hemorragia puede alterar su apariencia. La tortuosidad de la aorta puede desplazar esta banda.

\section{Ensanchamiento mediastinal}

Está definido como aumento del diámetro transverso del mediastino de más de $8 \mathrm{~cm}$ medido a la altura del arco aórtico ${ }^{20}$. Es uno de los signos más frecuentes de enfermedad mediastinal, presentándose en aproximadamente $77 \%$ de los pacientes con lesiones mediastinales ${ }^{13}$ (fig. 14).

Algunos autores consideran que la mejor forma de estimar ensanchamiento mediastinal (fig. 15) es el "ancho mediastinal izquierdo", el cual es una medida que se toma desde la línea media de la tráquea al borde lateral izquierdo del mediastino a nivel del arco aórtico. Sus valores normales son de 5 a $5,5 \mathrm{~cm}$. El ensanchamiento del "ancho mediastinal izquierdo" y del pedículo vascular puede presentarse también cuando hay dilatación de los grandes vasos, linfadenomegalias, masas mediastinales o alteraciones pleurales ${ }^{15,18}$.

Cuando se utilizan los valores de corte de 7,3-9,4cm, la especificidad para la detección de patologías mediastinales es alta. La sensibilidad de esta observación se incrementa cuando se usa el ancho mediastinal izquierdo ${ }^{21}$.

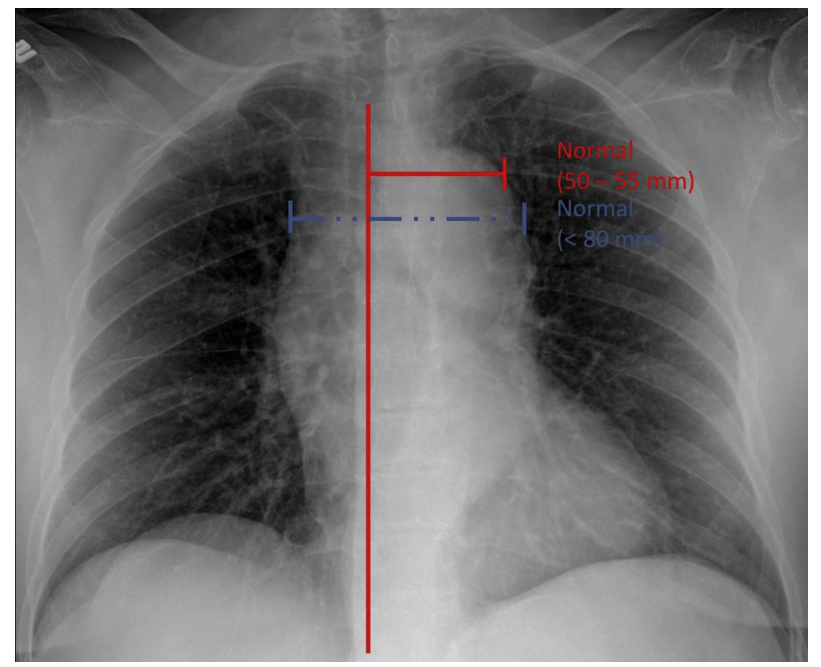

Figura 15 Medición del ancho mediastinal Medición del ancho mediastinal tradicional (líneas azules) y ancho mediastinal izquierdo (líneas rojas).

\section{Linfadenomegalias}

Son un hallazgo frecuente en muchas enfermedades mediastinales y su diagnóstico diferencial es amplio. Un alto porcentaje de las enfermedades que afectan el mediastino tiene dentro de su forma de presentación las linfadenomegalias, las cuales pueden o no estar asociadas con ensanchamiento mediastinal ${ }^{22}$. Las linfadenomegalias son la forma de presentación más frecuente del linfoma primario mediastinal, la extensión mediastinal del linfoma y el compromiso mediastinal por carcinoma broncogénico ${ }^{23}$ (fig. 16).

La sensibilidad y especificidad de la radiografía de tórax tiene un rendimiento moderado para la detección de adenomegalias, $67 \%$ y $59 \%$ respectivamente. Realizar en conjunto las proyecciones anteroposterior y lateral del tórax no incrementa la detección de adenomegalias ${ }^{24}$.

Las linfadenomegalias en linfoma, sarcoidosis y fibrosis quística son un hallazgo frecuente. En esta última son crónicas, no resuelven en el trascurso del tiempo y se asocian con mayor compromiso pulmonar ${ }^{25}$. 


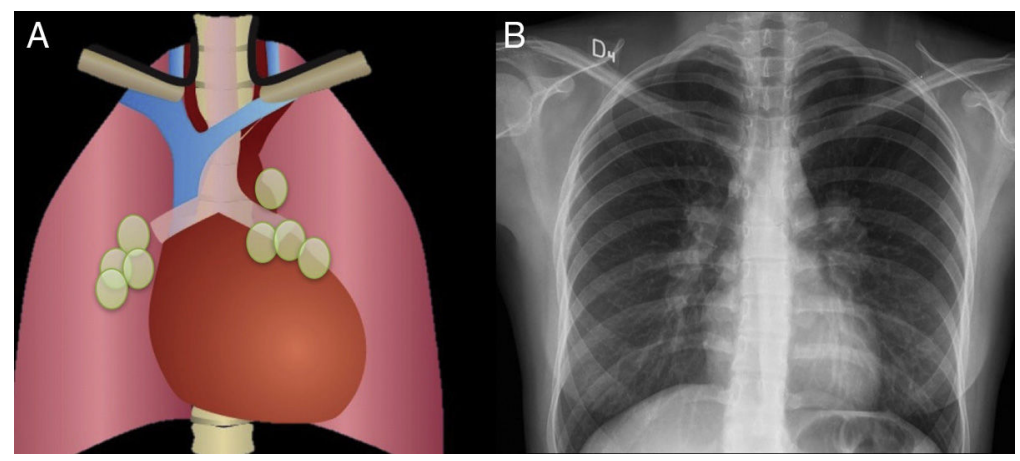

Figura 16 Linfadenomegalias

A. Esquema y B. Radiografía que muestra engrosamiento de ambas líneas paratraqueales por múltiples linfadenopatías que comprometen el mediastino medio. Se confirmó sarcoidosis.

\section{Desviación de la tráquea}

Es un signo muy útil en la detección de alteraciones mediastinales y es un hallazgo que orienta en una alteración del mediastino medio. En el paciente anciano hay una desviación fisiológica a la derecha del tercio inferior de la tráquea secundario al aplanamiento del arco aórtico y no debe ser confundido con una enfermedad ${ }^{14}$. La desviación de la tráquea también puede ser un hallazgo normal en la población pediátrica ${ }^{26}$.

Es, así mismo, un hallazgo incidental hasta en un $37 \%$ de los pacientes. En la práctica, este hallazgo no se correlaciona con dificultades en la intubación o aumento en la cantidad de intentos de intubación de estos pacientes ${ }^{26,27}$.

En el contexto de trauma, las alteraciones en la radiografía de tórax permiten sospechar lesión traqueal; sin embargo, tiene mayor sensibilidad para detectar lesiones del hilio pulmonar que lesiones de la tráquea ${ }^{28}$. Los hallazgos en la radiografía de tórax que sugieren una lesión traqueal son: enfisema cervical subcutáneo, neumomediastino, neumotórax y visualización de aire alrededor de un bronquio (fig. 7). También se debe sospechar lesión traqueal en las fracturas claviculares (del tercio proximal), escapulares, esternales o costales (primer y segundo arco) ${ }^{27,29,30}$

Pese a ser un hallazgo radiológico prominente, la desviación traqueal se encuentra más relacionada con enfermedades benignas, como el bocio intratorácico ${ }^{31}$. En las enfermedades malignas la desviación de la tráquea se asocia a linfadenomegalias ${ }^{32}$.

Las masas tiroideas suelen influir en la posición y las dimensiones de la tráquea debido a la proximidad de la tiroides con la pared traqueal y el hecho de que la cápsula tiroidea está entrelazada con el tejido conectivo que rodea la tráquea ${ }^{33}$ (fig. 17).

\section{Alteración en la interfaz de la arteria subclavia izquierda}

Es la interfaz que se forma entre la pleura del pulmón izquierdo, la arteria subclavia izquierda y la grasa mediastinal adyacente. Esta referencia mediastinal puede ser visualizada y alterarse ante linfadenomegalias, tortuosidad de la arteria subclavia o alteraciones pleurales.

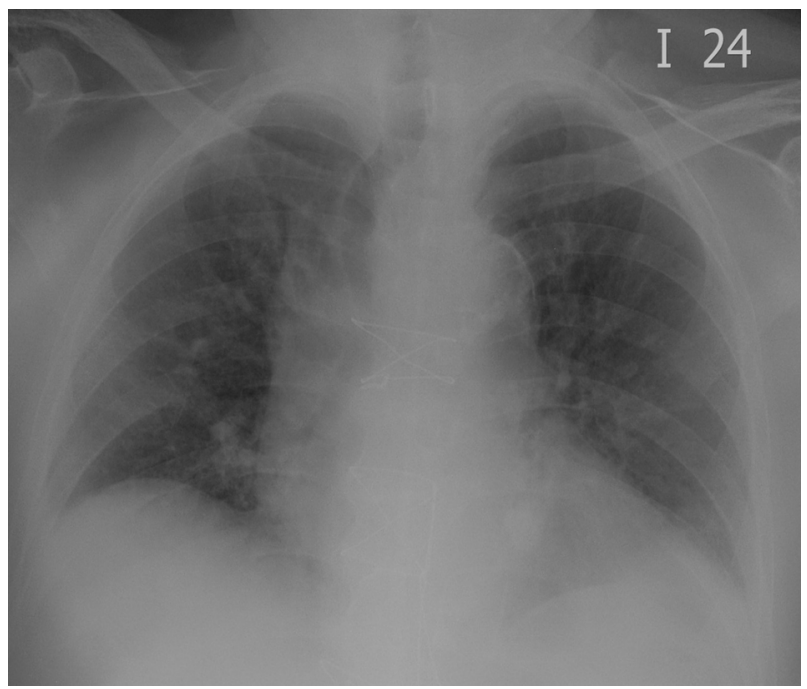

Figura 17 Desviación de la tráquea

Desviación significativa de la tráquea a la derecha, secundaria a aneurisma de la aorta ascendente. Opacidades alveolares bilaterales por neumonía multilobar.

\section{Mediastino posterior}

\section{Distorsión de las líneas paraespinales}

Las líneas paraespinales, también llamadas paravertebrales, se forman de la interfaz entre las porciones mediales de la pleura de los lóbulos posteriores y los tejidos paravertebrales de la columna (fig. 18). Son anormales cuando existe engrosamiento, desplazamiento o disrupción en su continuidad (tabla 6).

La línea paraespinal izquierda se encuentra por debajo del arco aórtico y se extiende verticalmente hasta la crura diafragmática izquierda paralela a la columna, en el medio de la columna vertebral y el margen lateral de la aorta. La línea paraespinal derecha es más delgada que la paravertebral izquierda y por tanto menos visible. La línea paraespinal derecha se visualiza mejor en su porción adyacente a las vértebras inferiores de la columna torácica, donde converge de forma vertical hacia la crura diafragmática derecha. En su 


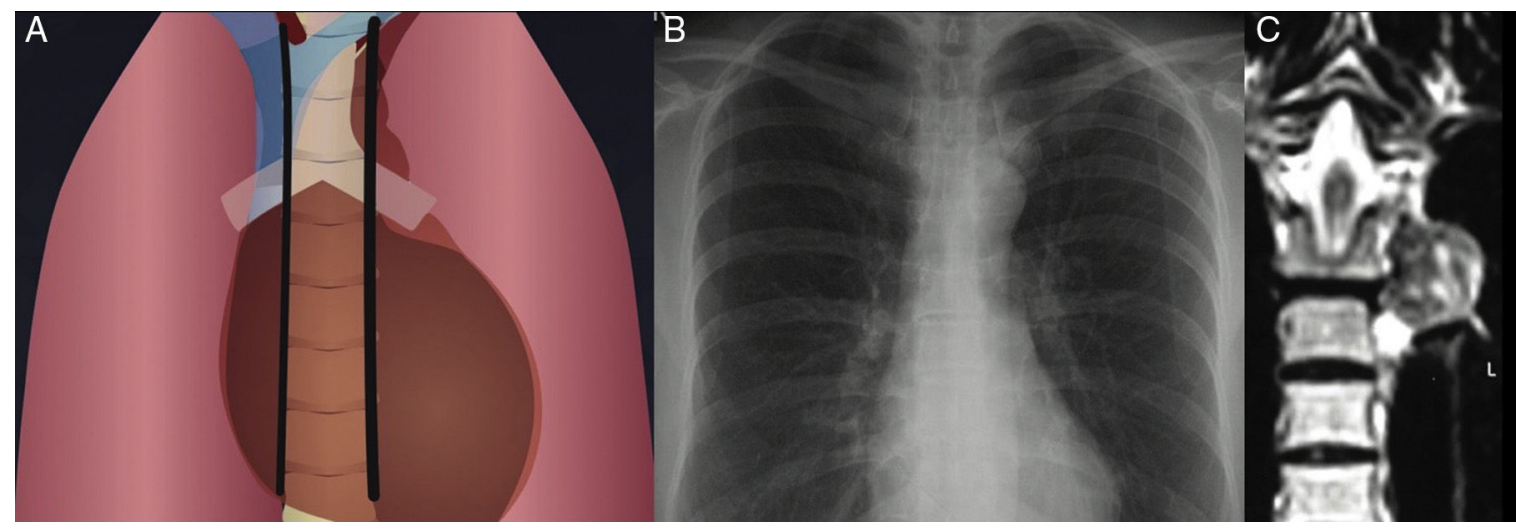

Figura 18 Líneas paraespinales

Ilustración (A) de las líneas paraspinales normales. La radiografía de tórax (B) demuestra una masa bien definida en el tercio superior del mediastino izquierdo, que se proyecta sobre el arco aórtico, originado de la línea paraspinal izquierda. La RM coronal con información T2 (C) muestra la misma masa paravertebral izquierda, que tiene una intensidad de señal heterogénea en un paciente con hematopoyesis extramedular. La visualización de la línea paraspinal izquierda es más frecuente que la línea derecha.

Tabla 6 Referencias del mediastino posterior

Distorsión de las líneas paraespinales

Distorsión de la línea para-aórtica

Obliteración de la línea de unión posterior

Distorsión del receso ácigo-esofágico

porción superior se visualiza adyacente a la columna de aire de la tráquea ${ }^{18}$.

La línea paravertebral izquierda se afecta usualmente por procesos que afectan el diámetro y recorrido de la aorta torácica. La causa más frecuente de engrosamiento de la línea paravertebral derecha son los osteofítos ${ }^{18}$. Las patologías que pueden afectar ambas líneas paravertebrales son: el trauma vertebral, el derrame pleural, las adenomegalias, las alteraciones vertebrales y paravertebrales, las masas paravertebrales posteriores y el ensanchamiento de las venas ácigos y hemiácigos.

En el contexto del trauma, las lesiones de las vértebras torácicas, particularmente las vértebras torácicas altas, se detectan a partir de la alteración de las líneas paraespinales y se acompañan de otras lesiones traumáticas mayores en el $83 \%$ de los casos (fig. 19) ${ }^{34}$. Por lo general, las vértebras torácicas altas son más difíciles de visualizar que el resto de los segmentos vertebrales dada la interposición de los hombros, las tres costillas superiores y el aumento de la densidad del mediastino superior. Por este motivo la detección de otros hallazgos que sugieran lesiones vertebrales traumáticas es muy útil. Estos hallazgos incluyen el desplazamiento de los cuerpos vertebrales, de los procesos espinosos y de la sonda nasogástrica, así como la pérdida de la altura de los cuerpos vertebrales, la alteración en la forma de las facetas articulares y el ensachamiento mediastinal ${ }^{35}$.

\section{Distorsión de la línea paraaórtica}

Es una línea que se visualiza mejor en la proyección lateral. Esta línea se produce por el contacto entre la aorta descendente y el aspecto medial del lóbulo inferior izquierdo.
Se observa por debajo el arco aórtico, paralela a la línea paravertebral izquierda. Su visualización en las radiografías de tórax es frecuente. Se puede acentuar ante los casos de hipercifosis dorsal, tortuosidad de la aorta y enfisema pulmonar ${ }^{14}$.

El desplazamiento del arco aórtico en más de $10 \mathrm{~mm}$, desde la media de la circunferencia aórtica al margen izquierdo de la tráquea, tiene sensibilidad y especificidad moderadas, $78 \%$ para cada una, en el diagnóstico de una masa mediastinal esofágica paratraqueal izquierda ${ }^{35}$ (fig. 20).

La obliteración de la línea paraaórtica puede darse sin que represente necesariamente una condición patológica ${ }^{36}$ y puede explicarse por la aposición de cualesquiera de las estructuras hiliares, incluida la vena pulmonar izquierda, la grasa mediastinal y el ventrículo izquierdo.

El aumento de su convexidad se relaciona con tortuosidad o dilatación de la aorta. Ocasionalmente se pueden observar convexidades focales de la línea paraaórtica que pueden corresponder a aneurismas aórticos, adenomegalias, masas paraespinales o tumores neurogénicos ${ }^{14}$.

\section{Obliteración de la línea de unión posterior}

Se forma por la aposición de la pleura y una cantidad variable de grasa en el mediastino superior, posterior al esófago y anterior a los cuerpos vertebrales torácicos superiores. Usualmente es una línea recta o levemente cóncava dirigida en sentido caudal que siempre termina en el borde superior del arco aórtico. Es frecuente una referencia mediastinal que se visualiza comúnmente y se puede alterar ente enfermedades o masas esofágicas ${ }^{18}$ (fig. 21).

\section{Distorsión del receso ácigo-esofágico}

El receso ácigo-esofágico es una interfaz mediastinal generada por el espacio entre la pared lateral del tercio medio e inferior del esófago y las pleuras pulmonares anteriores a la columna. Se extiende desde el cayado de la vena ácigos hasta el hiato aórtico ${ }^{2}$. La línea ácigo-esofágica se 


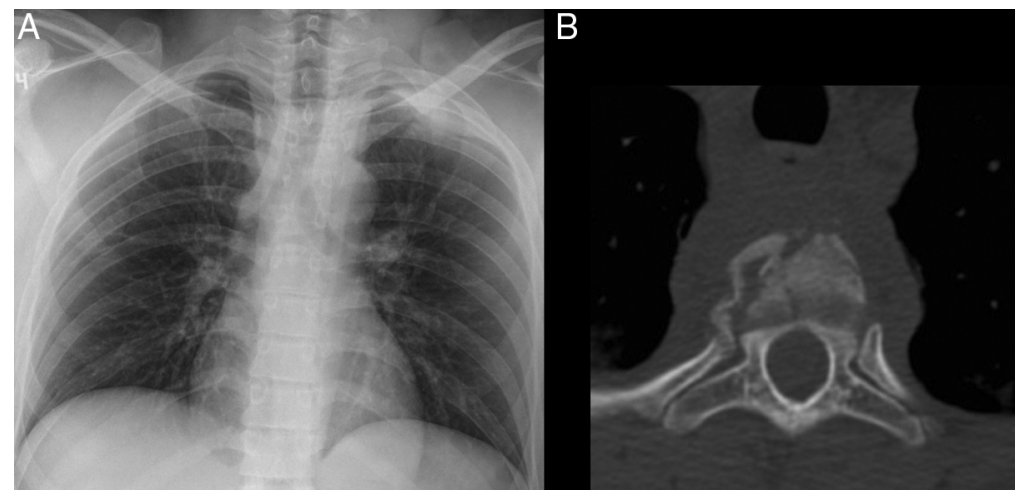

Figura 19 Fractura vertebral y relación con las líneas paraespinales

Paciente de 23 años con antecedentes de trauma. (A) Radiografía de tórax mostrando ensanchamiento de las líneas paraspinales en el tercio superior del tórax sin una clara disminución o alteración de los cuerpos vertebrales. En la tomografía de tórax (B) se observa una fractura impactada del cuerpo vertebral de T3 con prominencia de tejido prevertebral y paravertebral que corresponde a hematoma.

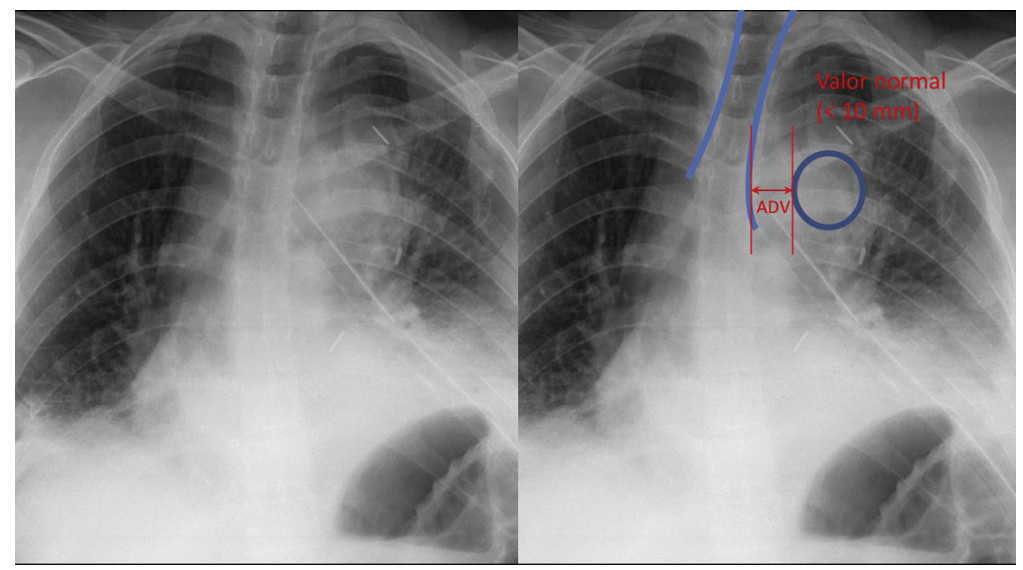

Figura 20 Desplazamiento del botón aórtico

Desplazamiento del botón aórtico en la radiografía de tórax; Dibujamos un círculo verdadero a lo largo de la botón aórtico. La magnitud del desplazamiento aórtico se mide como la distancia entre el borde medial del círculo y el margen izquierdo de la columna aérea traqueal. En este paciente se observa un ADV (valor de desplazamiento aórtico) superior a $10 \mathrm{~mm}$. Se confirmó hemorragia mediastinal.

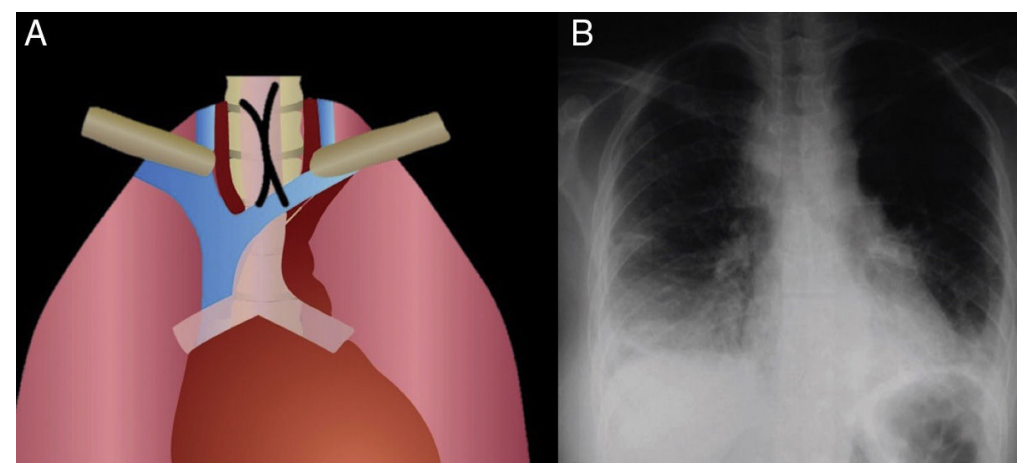

Figura 21 Obliteración de la línea de unión posterior

A. Esquema mostrando la línea de unión posterior. B. Ensanchamiento mediastínal, derrames pleurales bilaterales y ausencia de línea de unión posterior en un paciente con absceso por mediastinitis retrofaríngea. 


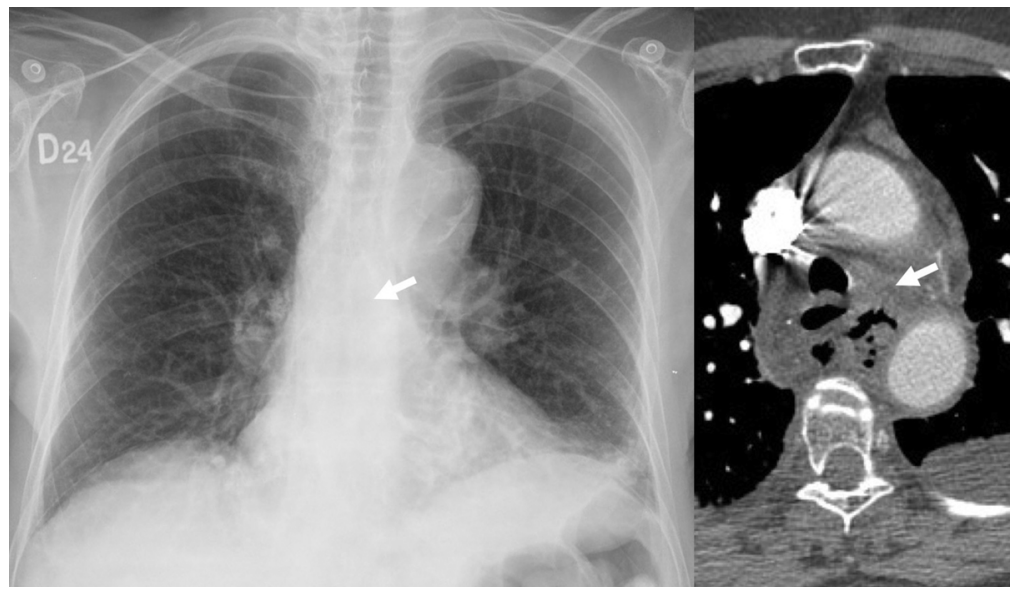

Figura 22 Distorsión del receso azigo esofágico

Rectificación y desviación del receso azigo esofágico posterior, relacionado con proceso de mediastinitis y absceso mediastinal.

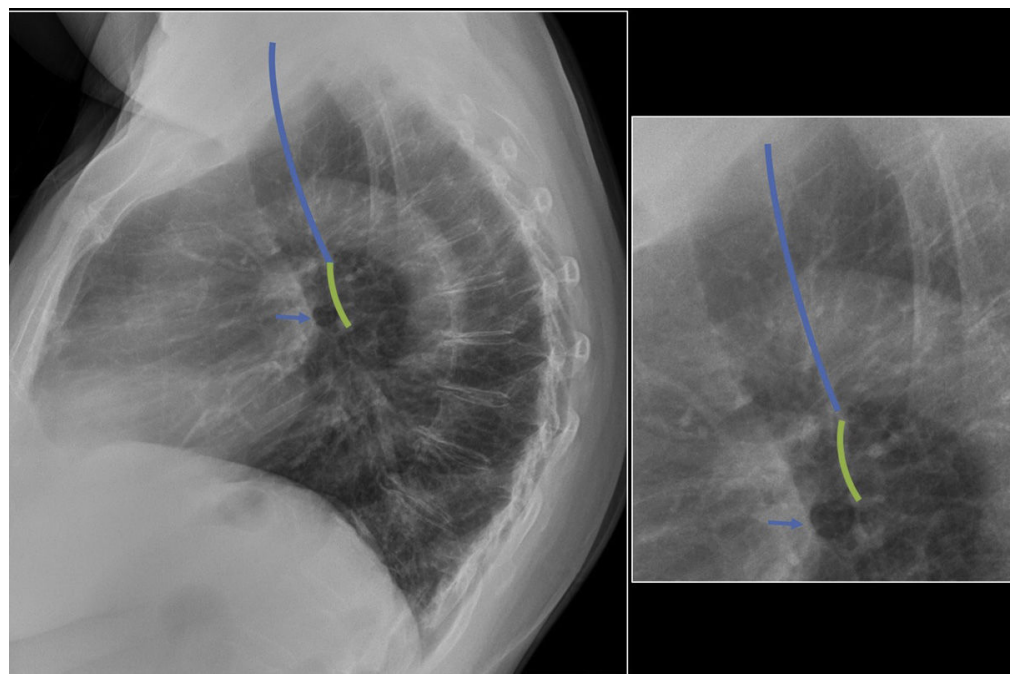

Figura 23 Radiografía lateral de la anatomía hiliar

Radiografía lateral que muestra la anatomía hiliar parcial. La pared posterior de la tráquea (línea azul) es visible en la parte superior. El bronquio del lóbulo superior derecho (flecha azul) puede ser visto como una lucencia ovalada dentro de la sombra hiliar superior. Debajo de la pared posterior de la tráquea, la pared posterior del bronquio intermedio (línea verde) es visible al nivel de su bifurcación.

visualiza como una "S invertida". Tiene una leve convexidad hacia la izquierda en el segmento superior con un borde recto inferior. La convexidad superior derecha puede verse en niños y adultos jóvenes, pero es anormal en adultos mayores ${ }^{2,37}$. Puede presentarse con morfología cóncava en el $21 \%$ de los pacientes sin que esto represente alteraciones $^{38}$ (fig. 22).

El desplazamiento de la línea ácigo-esofágica puede surgir de atelectasias parciales o completas de los lóbulos inferiores, en la dilatación esofágica y en las masas mediastinales posteriores ${ }^{37}$. Un contorno anormal de esta línea puede deberse a linfadenopatía, hernias hiatales, malformaciones broncopulmonares, neoplasias esofágicas, anormalidades pleurales y cardiomegalia con dilatación de la aurícula izquierda².

\section{Mediastino en proyección lateral}

\section{Línea traqueal posterior}

Se forma por la aposición de la pared posterior de la tráquea, los tejidos blandos y la pleura del pulmón derecho, y tiene un grosor normal de $2,5 \mathrm{~mm}$. En algunos pacientes puede tener mayor grosor y alcanzar hasta $5,5 \mathrm{~mm}$. En ese caso se la denomina banda traqueal posterior y es un hallazgo que se 
explica por la interfaz de la pared posterior de la tráquea con la pared anterior del esófago, en lugar del pulmón derecho ${ }^{39}$ (fig. 23).

\section{Pared posterior del bronquio intermedio}

Es una línea vertical que corresponde en su porción superior a la pared posterior del bronquio principal derecho y en su porción inferior a la pared posterior del bronquio intermedio (fig. 23) 39,40 .

La pared posterior del bronquio intermedio normalmente tiene un grosor de $0,5-2 \mathrm{~mm}$ y se considera un engrosamiento anormal, cuando se encuentra por encima de $3 \mathrm{~mm}$. Su longitud máxima es de $50 \mathrm{~mm}$. Solo logra delinearse adecuadamente en el $55 \%$ de los pacientes ${ }^{40-42}$ (fig. 23).

\section{Conclusiones}

Las lesiones mediastinales suelen presentarse de manera inespecífica y a pesar de las técnicas en biopsia y avances en imágenes se requiere un examen histológico para estabelecer un diagnóstico y descartar malignidad.

Por tradición, se han mencionado referencias anatómicas como líneas, bandas e interfaces, las cuales son de utilidad en la identificación de lesiones mediastinales, sin embargo, hasta la fecha, dichas referencias tienen porcentajes de visualización variables y no logran una sensibilidad suficientemente alta para orientar hacia un diagnóstico etiológico preciso de una masa mediastinal; sin embargo, existen características mucho más sensibles para alertar sobre la presencia de lesiones mediastinales como son el ensanchamiento mediastinal y adenomegalias.

Con un abordaje cuidadoso de las lesiones mediastinales a partir de la semiología radiológica y el énfasis en la prevalencia de las lesiones mediastinales en cada compartimiento, se logra acortar los diagnósticos diferenciales.

\section{Financiación}

Ninguna.

\section{Conflicto de interés}

Ninguno.

\section{Agradecimientos}

A todo el equipo de Radiología torácica del Hospital Universitario de la Fundación Santa Fe de Bogotá.

\section{Bibliografía}

1. Proto AV. Mediastinal anatomy: emphasis on conventional images with anatomic and computed tomographic correlations. J Thorac Imaging. 1987;2:1-48.

2. Gibbs JM, Chandrasekhar CA, Ferguson EC, Oldham SAA. Lines and stripes: where did they go? -From Conventional Radiography to CT. RadioGraphics. 2007;27:33-48.

3. Heitzman ER. The Mediastinum: radiologic correlations with anatomy and pathology. 2 nd. ed. 1988. Softcover reprint of the original 2 nd. ed. 1988 edition. Berlin, Heidelberg: Springer; 2012. p. 355.

4. Panikkath R, Panikkath D. Mach band sign: an optical illusion. Proc Bayl Univ Med Cent. 2014;27:364-5.

5. Chasen MH. Practical applications of mach band theory in thoracic analysis. Radiology. 2001;219:596-610.

6. Pearls and Pitfalls in Emergency Radiology: Variants and Other Difficult Diagnoses: 9781107021914: Medicine \& Health Science Books @ Amazon.com [Internet]. [Acceso 22 Sep 2017]. Disponible en: https://www.amazon.com/ Pearls-Pitfalls-Emergency-Radiology-Difficult/dp/110702191X.

7. Mcminn. Last's Anatomy: Regional and Applied. Australia: Elsevier; 2003.

8. Zylak CJ, Pallie W, Pirani M, Wandtke JC, Kothari K. Anatomy and computed tomography. RadioGraphics. 1983;3:478-530.

9. Fraser RS, Müller NL, Colman N, Paré PD. Fraser and Paré's diagnosis of diseases of the chest. Volumes 1-4. Fraser Parés Diagn Dis Chest Vol 1-4 [Internet]. 1999 [Acceso 24 Sep 2017]; Disponible en: https://www.cabdirect.org/ cabdirect/abstract/20002009269.

10. Fujimoto K, Hara M, Tomiyama N, Kusumoto M, Sakai F, Fujii Y. Proposal for a new mediastinal compartment classification of transverse plane images according to the Japanese Association for Research on the Thymus (JART) General Rules for the Study of Mediastinal Tumors. Oncol Rep. 2014;31: $565-72$.

11. Carter BW, Tomiyama N, Bhora FY, Rosado de Christenson ML, Nakajima J, Boiselle PM, et al. A Modern Definition of Mediastinal Compartments. J Thorac Oncol. 2014;9:S97-101.

12. Takeda S, Miyoshi S, Akashi A, Ohta M, Minami M, Okumura M, et al. Clinical spectrum of primary mediastinal tumors: a comparison of adult and pediatric populations at a single Japanese institution. J Surg Oncol. 2003;83:24-30.

13. Aroor AR. A Study of Clinical Characteristics of Mediastinal Mass. J Clin Diagn Res [Internet]. 2014 [Acceso 7 Oct 2014]; Disponible en: http://www-ncbi-nlm-nih-gov. ezproxy.uniandes.edu.co:8080/pmc/articles/PMC3972605/.

14. Coche EE, Ghaye B, Mey JD, Duyck P. Comparative interpretation of CT and standard radiography of the chest. Springer Science \& Business Media; 2011.

15. Giron J, Fajadet P, Sans N, Jarlaud T, Verhnet H, Galy-Fourcade $D$, et al. Diagnostic approach to mediastinal masses. Eur J Radiol. 1998;27:21-42.

16. McComb BL. Reflecting upon the left superior mediastinum. J Thorac Imaging. 2001;16:56-64.

17. Wright FW. Radiology of the chest and related conditions. CRC Press; 2001.

18. Webb WR, Higgins CB. Thoracic imaging: pulmonary and cardiovascular radiology. Lippincott Williams \& Wilkins; 2010.

19. Hansell DM. Imaging of Diseases of the Chest: Expert Consult Online and Print. $5^{\text {th }}$. ed. Edinburgh: Mosby; 2009.

20. Lai V, Tsang WK, Chan WC, Yeung TW. Diagnostic accuracy of mediastinal width measurement on posteroanterior and anteroposterior chest radiographs in the depiction of acute nontraumatic thoracic aortic dissection. Emerg Radiol. 2012;19:309-15.

21. Lai V, Tsang WK, Chan WC, Yeung TW. Diagnostic accuracy of mediastinal width measurement on posteroanterior and anteroposterior chest radiographs in the depiction of acute nontraumatic thoracic aortic dissection. Emerg Radiol. 2012;19:309-15.

22. Naidich DP, Müller NL, Webb WR. Computed Tomography and Magnetic Resonance of the Thorax. Lippincott Williams \& Wilkins; 2007.

23. Suwatanapongched T, Gierada DS. CT of thoracic lymph nodes. Part II: diseases and pitfalls. Br J Radiol. 2006;79:999-1000.

24. Swingler G, du Toit G, Andronikou S, van der Merwe L, Zar $\mathrm{H}$. Diagnostic accuracy of chest radiography in detecting 
mediastinal lymphadenopathy in suspected pulmonary tuberculosis. Arch Dis Child. 2005;90:1153-6.

25. Don CJ, Dales RE, Desmarais RL, Neimatullah M. The radiographic prevalence of hilar and mediastinal adenopathy in adult cystic fibrosis. Can Assoc Radiol J J Assoc Can Radiol. 1997;48:265-9.

26. Costa NS, Laor T, Donnelly LF. Superior cervical extension of the thymus: a normal finding that should not be mistaken for a mass. Radiology. 2010;256:238-42.

27. Kar P, Rath GP, Prabhakar H, Ali Z. Tracheal deviation may be a normal anatomical variant in children. Anaesth Intensive Care. 2009;37:144-5.

28. Scaglione M, Romano S, Pinto A, Sparano A, Scialpi M, Rotondo A. Acute tracheobronchial injuries: Impact of imaging on diagnosis and management implications. Eur J Radiol. 2006;59:336-43.

29. Unger JM, Schuchmann GG, Grossman JE, Pellett JR. Tears of the trachea and main bronchi caused by blunt trauma: radiologic findings. AJR Am J Roentgenol. 1989;153:1175-80.

30. Hong BW, Mazeh H, Chen H, Sippel RS. Routine Chest X-ray prior to thyroid surgery: is it always necessary? World J Surg. 2012;36:2584-9.

31. Adegboye VO, Brimmo Al, Adebo OA, Ogunseyinde 00, Obajimi MO. The place of clinical features and standard chest radiography in evaluation of mediastinal masses. West Afr J Med. 2003;22:156-60.

32. Díez JJ. Goiter in adult patients aged 55 years and older: etiology and clinical features in 634 patients. J Gerontol A Biol Sci Med Sci. 2005;60:920-3.

33. Buckley JA, Stark P. Intrathoracic mediastinal thyroid goiter: imaging manifestations. AJR Am J Roentgenol. 1999;173:471-5.

34. van Beek EJR, Been HD, Ponsen K-J, Maas M. Upper thoracic spinal fractures in trauma patients - a diagnostic pitfall. Injury. 2000;31:219-23.
35. Yang DH, Seo JB, Lee IS, Do K-H, Ko SM, Lee S-H, et al. Displaced aortic arch sign on chest radiographs: a new sign for the detection of a left paratracheal esophageal mass. Eur Radiol. 2004;15:936-40.

36. Takahashi K, Shinozaki T, Hyodo H, Ogawa C, Ohsawa T. Focal obliteration of the descending aortic interface on normal frontal chest radiographs: correlation with CT findings. Radiology. 1994;191:685-90.

37. Marchiori D. Clinical Imaging: With Skeletal, Chest and Abdomen Pattern Differentials. Elsevier Health Sciences; 2004.

38. Heitzman ER, Groskin SA. The Lung: Radiologic-pathologic Correlations. $3^{\text {rd }}$. Ed. St. Louis: Mosby; 1993.

39. Franquet T, Erasmus JJ, Giménez A, Rossi S, Prats R. The retrotracheal space: normal anatomic and pathologic appearances. Radiogr Rev Publ Radiol Soc N Am Inc. 2002, 22 Spec No: S231-46.

40. Webb WR, Hirji M, Gamsu G. Posterior wall of the bronchus intermedius: radiographic-CT correlation. AJR Am J Roentgenol. 1984;142:907-11.

41. Frija J, de Kerviler E, Zagdanski AM. Radiologic anatomy of the inferior lung margins as demonstrated on computed radiography with enhancement of low frequencies. Surg Radiol Anat. 1997; 19:257-63.

42. Biemans JMA, Van Heesewijk JPM, Van Der Graaf Y. Digital chest imaging: selenium radiography versus storage phosphor imaging. Comparison of visualization of specific anatomic regions of the chest. Invest Radiol. 2002;37:47-51.

43. Dobson MJ, Carrington BM, Parsons VJ, Lo F, Coffey J, Ryder $W D$, et al. What is the value of the lateral chest radiograph in the follow-up thoracic lymphoma? Eur Radiol. 1997;7: 1110-3. 